\title{
Evaluation of Parents and Hybrids for Yield and Quality characters in Bitter Gourd (Momordica charantia L.)
}

\author{
K. Mallikarjunarao ${ }^{1 *}$, A.K. Das ${ }^{1}$, A. Nandi $^{1}$, B. Baisakh ${ }^{2}$, G.S. Sahu ${ }^{1}$ and P. Tripathy ${ }^{1}$ \\ ${ }^{1}$ Department of Vegetable Science, O.U.A.T. Bhubaneswar-751003 Odisha \\ ${ }^{2}$ Dean, PGF-CUM-DRI, O.U.A.T. Bhubaneswar-751003 Odisha \\ *Corresponding author
}

\begin{abstract}
A B S T R A C T
Keywords

Bitter Gourd, hybrids, yield and quality characters

Article Info

Accepted:

08 July 2018

Available Online:

10 August 2018

The experiment was conducted at AICRP on vegetable crops, OUAT, Bhubaneswar, India to study the performance of twenty eight bitter gourd hybrids along with eight genetically diverse parents. The analysis of variance for experimental design revealed that the differences among the mean square due to treatments were found highly significant for all the traits. Further variance due to parents and hybrids (within group) were also found significant for all the studied characters. Difference due to parents Vs hybrids were also found highly significant for all characters studied. The parents Phule Green Gold, Improved Katahi and Preethi were observed to be top performing parent for fruit yield per vine and Thusi for number of primary branches per vine and number of fruit per vine. The hybrids Phule Green Gold x Pusa Do Mausami, Phule Green Gold x Preethi and Preethi x Pusa Do Mausami were recorded to be three best performing $F_{1}$ hybrids for fruit yield per vine with an yield of $3.13,3.00$ and $2.75 \mathrm{~kg}$, respectively. These top performing $\mathrm{F}_{1}$ hybrids can be tested in different seasons over different locations for assessing their stability for high yield.
\end{abstract}

\section{Introduction}

Momordica charantia usually reffered to as bitter gourd may be a tropical and subtropical valuable commercial vegetable crop of the family Cucurbitaceae. Bitter gourd has been utilized in numerous herbal medicine systems for a long time because of its malady preventing and health promoting phyto chemical compounds like dietary fiber, minerals, vitamins, flavonoids and antioxidants. Consumption of its fruit juice is extremely helpful for diabetic patients due to its potent oxygen free radical scavenging activity (Sreejayan and Rao, 1991). Fruits and seeds of bitter gourd are consumed together at immature stage and both possess therapeutic properties such as anti-diabetes (Chen et al., 2003), anti-carcinogenic and hypercholesterolemic (Ganguly et al., 2000; Ahmed et al., 2001), charantin (Yeh et al., 2003), hypoglycemic compounds (Jayasooriya et al., 2000), momorcharin (inactivating ribosome; Leung et al., 1997), MAP30 (a momordica-HIV protein that suppress HIV activity; Lee-Huang et al., 1995), 
momordicoside A and B (tumour growth inhibitor; Okabe et al., 1980). The fruits of bitter gourd additionally possess anti- fertility (Basch et al., 2003), anti-microbial (Yesilada et al., 1999), anti-viral (Nerurkar et al., 2006), and anti-ulcerogenic (Gurbuz et al., 2000), anti-tumour (Taylor, 2002) activities characterized in bitter gourd attributed to broad array of biologically active phytochemicals together with triterpenes and steroids (Grovar and Yadav, 2004). The seeds of bitter gourd contain pyrimidine nucleoside vicine (Dutta et al., 1981 and Barron et al., 1982). Throughout the past decade the antidiabetic properties of the crop are studied broadly and a hypoglycaemic principle called charantin has been isolated. The bitterness of bitter gourd is due to the cucurbitacin like alkaloid momordicine and triterpene glycosides viz., momordicoside $\mathrm{K}$ and $\mathrm{L}$ (Jeffrey, 1980 and Okabe et al.,1982). Leading states cultivating bitter gourd in India are Tamil Nadu, Kerala, Maharashtra, Uttar Pradesh, Andhra Pradesh, Gujarat etc. and India produces about nine lakh metric tons of bitter gourd fruit from an area of 98,300 hectare (NHB database, 2015-16). Even though India is major producer of bitter gourd in the world, the normal productivity is extremely low and becomes static over the decades. Major constraints to productivity of bitter gourd are low genetic potential of improved varieties, lack of early and high yielding hybrids, and inaccessibility of quality seeds besides poor crop management, biotic and abiotic stresses. Hybrids in most of the vegetable crops offer opportunity of earliness, high yield, and quality improvement besides better capability to counteract biotic and abiotic stresses. The hybrid in bitter gourd may be constrained because of conventional practice of hand pollination, which requires lot of labour and time. There is a prime requiring for its enhancement and to create varieties or hybrids suited to particular agro ecological conditions. Hence, in the present investigation, parents and hybrids were evaluated to identify the best parents and hybrid combination.

\section{Materials and Methods}

The study was taken up during summer season of 2016 at AICRP on vegetable crops, OUAT, Bhubaneswar. The experimental material consisted of eight genetically diverse inbred lines of bitter gourd viz., Phule Green Gold, Improved Katahi, Hirkani, CO-1, Nakhara Local, Thusi, Preethi and Pusa Do Mausami together with twenty eight $F_{1}$ hybrids obtained through half diallel mating among the selected parents. The crop was planted in rows spaced at 1.5 meters with plant to plant spacing of 1.0 meter in a randomised block design with three replications. Observations were recorded from four randomly selected plants on quantitative and qualititative traits viz., vine length $(\mathrm{m})$, number of primary branches per vine, internodal length $(\mathrm{cm})$, fruit length $(\mathrm{cm})$, fruit diameter $(\mathrm{cm})$, average fruit weight $(\mathrm{g})$, number of fruits per vine, fruit yield per vine (kg), flesh thickness (mm), TSS ( ${ }^{\circ}$ Brix) and vitamin $\mathrm{C}$ content $(\mathrm{mg} / 100 \mathrm{~g})$. The mean data was subjected to statistical analysis as suggested by Panse and Sukathme (1957).

\section{Results and Discussion}

Analysis of variance for eight parents and twenty eight hybrids indicated powerful significant variability among them for all the characters (Table 1). This shows the existence of a noticeable amount of variability in the base material as well as in the generated materials, fulfilling the fundamental prerequisite for considering the genotypes further. Comparison of mean squares due to parents Vs hybrids was found to be significant for all traits. The mean values of $F_{1}$ hybrids were higher than those of parents (Table 2) for all the characters studied, which shown plentiful scope for the improvement of these characters. In any crop breeding programme, it 
is fundamental to remove the undesirable types, which can be accomplished by studying the mean performance of parents and hybrids. Per se performance should be given an equal importance while judging the hybrid combinations for exploitation of heterosis.

Vine length is an essential yield component by which growth and vigour of plants are measured. In the present study vine length was high in the parents Preethi $(4.40 \mathrm{~m})$ followed by CO-1 $(4.25 \mathrm{~m})$ and Phule Green Gold (4.08 $\mathrm{m})$ and the hybrids Phule Green Gold $\mathrm{x}$ Preethi shows highest vine length of $(4.90 \mathrm{~m})$ followed by Phule Green Gold x Pusa Do Mausami $(4.86 \mathrm{~m})$ and Preethi x Pusa Do Mausami $(4.69 \mathrm{~m})$ exhibited high vine length. The parent Thusi (2.94) and the hybrids Improved Katahi x Hirkani (3.12 m), Nakhara Local $\mathrm{x}$ Thusi $(3.30 \mathrm{~m})$ and CO-1 $\mathrm{x}$ Thusi $(3.30 \mathrm{~m})$ were shortest. The same patterns of results in their study were noted by Rajeswari (1998) and Sundaram (2006).

In the present study number of primary branches per vine was high in the parents, Thusi (16.75) and the hybrids, Thusi $x$ Pusa Do Mausami (19.50), Hirkani x Thusi (19.00) appeared most noteworthy number of primary branches per vine, whereas the parent $\mathrm{CO}-1$ (8.75) and the hybrid CO-1 x Nakhara Local (7.92) appeared least number of primary branches per vine. Lower intermodal length is the favorable trait in cucurbits. Within the present study the parents Pusa Do Mausami $(4.25 \mathrm{~cm})$, Thusi $(4.43 \mathrm{~cm})$ and Nakahara Local $(4.50 \mathrm{~cm})$, and the hybrid combinations Hirkani x Thusi $(4.00 \mathrm{~cm})$, Thusi $\mathrm{x}$ Pusa Do Mausami $(4.04 \mathrm{~cm})$ and Improved Katahi $x$ Thusi $(4.10 \mathrm{~cm})$ had the lower intermodal length. The same pattern of results in their study was described by Rajeswari (1998), Sundaram (2006) and Rani et al., (2014).

Fruit length and diameter are also imperative traits to decide yield besides market preference, purpose of use and means of handling. Longest fruit was found in parent, Phule Green Gold (18.04 cm), CO-1 (14.15 $\mathrm{cm})$ and Preethi $(13.34 \mathrm{~cm})$ whereas, in hybrids Phule Green Gold $x$ Pusa Do Mausami $(22.68 \mathrm{~cm})$, Phule Green Gold x CO-1 $(19.50 \mathrm{~cm})$ and Phule Green Gold $\mathrm{x}$ Preethi $(19.00 \mathrm{~cm})$ recorded higher fruit length, in this way it was evident that, the parents with longer fruits were effectively involved in producing hybrids with long fruits (Laxuman et al., 2012). From point of view customer approval, the perfect fruit diameter should be between 4 to $5 \mathrm{~cm}$. From this point of view 50 per cent of hybrids evaluated have sufficient fruit diameter. For trait fruit diameter, most noteworthy fruit diameter was found in parent Preethi $(4.72 \mathrm{~cm})$ and Improved Katahi $(4.19 \mathrm{~cm})$, although among hybrids Improved Katahi x Preethi had more fruit diameter of $(5.20 \mathrm{~cm})$ taken after by Preethi x Pusa Do Mausami $(5.15 \mathrm{~cm})$, Phule Green Gold x Preethi $(5.00 \mathrm{~cm})$.

Numbers of fruits and fruit weight specifically decide bitter gourd yield (Dey et al., 2005). Among the parents average fruit weight was highest in Preethi (83.98 g), Phule Green Gold (71.35 gm) and CO-1 (63.48 g) and among the hybrids Phule Green Gold $x$ Pusa Do Mausami (103.53 g), Phule Green Gold x Preethi $(102.40 \mathrm{~g})$ and Preethi $\mathrm{x}$ Pusa Do Mausami $(99.62 \mathrm{~g})$ recorded higher fruit weight. Based on the mean performance, the hybrids Thusi x Pusa Do Mausami (41.92), Hirkani x Thusi (39.83) and Improved Katahi $x$ Thusi (35.75) were producing higher number of fruits per vine. The parents Thusi (36.17), Hirkani (30.25) and Improved Katahi (29.42) were found to be the best for this character. The same trend of results was recorded by Devadas (1993). The more number of fruits in Thusi might be ascribed due to more number of primary branches. These traits possibly boost the number of leaves and subsequently the photosynthetic efficiency. Comparative results were recorded by Harika et al., (2012) in bottle gourd. 
Table.1 Analysis of variance (Mean squares) for various characters in bitter gourd during summer season -2016

\begin{tabular}{|c|c|c|c|c|c|c|c|c|c|}
\hline Source & d.f & $\begin{array}{l}\text { Vine } \\
\text { length } \\
(\mathrm{m})\end{array}$ & $\begin{array}{c}\text { Number of } \\
\text { primary } \\
\text { branches per } \\
\text { vine }\end{array}$ & $\begin{array}{l}\text { Internodal } \\
\text { length (cm) }\end{array}$ & $\begin{array}{l}\text { Fruit length } \\
\qquad(\mathrm{cm})\end{array}$ & $\begin{array}{l}\text { Fruit } \\
\text { diameter } \\
(\mathbf{c m})\end{array}$ & $\begin{array}{c}\text { Average } \\
\text { fruit } \\
\text { weight (g) }\end{array}$ & $\begin{array}{l}\text { Number of } \\
\text { fruits per } \\
\text { vine }\end{array}$ & $\begin{array}{c}\text { Fruit yield } \\
\text { per vine } \\
\text { (kg) }\end{array}$ \\
\hline Replications & 2 & 0.05 & 0.58 & 0.01 & 3.72 & 0.01 & 7.34 & 3.16 & 0.06 \\
\hline Treatments & 35 & $0.89 * *$ & $22.97 * *$ & $3.79 * *$ & $34.11 * *$ & $0.87 * *$ & $1358.05^{* *}$ & $96.28 * *$ & $1.35 * *$ \\
\hline Parents & 7 & $1.04 * *$ & $20.80 * *$ & $2.14 * *$ & $44.83 * *$ & $0.69 * *$ & $1303.22 * *$ & $104.36 * *$ & $0.45 * *$ \\
\hline Hybrids & 27 & $0.84 * *$ & $23.21 * *$ & $4.11 * *$ & $30.40 * *$ & $0.90 * *$ & $1342.89 * *$ & $95.41 * *$ & $1.47 * *$ \\
\hline Parents Vs Hybrids & 1 & $1.30 * *$ & $31.72 * *$ & $6.84 * *$ & $59.33 * *$ & $1.42 * *$ & $2151.15^{* *}$ & $63.51 * *$ & $4.41 * *$ \\
\hline Error & 70 & 0.03 & 1.55 & 0.04 & 1.25 & 0.03 & 9.34 & 5.27 & 0.02 \\
\hline
\end{tabular}

$*$, ** significant at $5 \%$ and $1 \%$ level, respectively

Table 1. contd....

\begin{tabular}{|c|c|c|c|c|}
\hline Source & d.f & $\begin{array}{c}\text { Flesh thickness } \\
(\mathbf{m m})\end{array}$ & TSS $\mathbf{(}^{\mathbf{0} \text { Brix) }}$ & $\begin{array}{c}\text { Vitamin C } \\
(\mathbf{m g} / \mathbf{1 0 0 g})\end{array}$ \\
\hline Replications & 2 & 0.02 & 0.06 & 43.79 \\
\hline Treatments & 35 & $9.81^{* *}$ & $1.17 * *$ & $843.08^{* *}$ \\
\hline Parents & 7 & $6.45^{* *}$ & $0.85^{* *}$ & $343.63^{* *}$ \\
\hline Hybrids & 27 & $10.35^{* *}$ & $1.24 * *$ & $959.67 * *$ \\
\hline $\begin{array}{c}\text { Parents Vs } \\
\text { Hybrids }\end{array}$ & 1 & $18.73^{* *}$ & $1.38^{* *}$ & $1191.47^{* *}$ \\
\hline Error & 70 & 0.05 & 0.06 & 61.38 \\
\hline
\end{tabular}

$*$,** significant at $5 \%$ and $1 \%$ level, respectively

\footnotetext{
, *
} 
Table.2 Mean performance of parents and hybrids for yield and its contributing traits during summer season - 2016

\begin{tabular}{|c|c|c|c|c|c|c|}
\hline Hybrid & $\begin{array}{l}\text { Vine length } \\
\text { (m) }\end{array}$ & $\begin{array}{c}\text { Number of } \\
\text { primary } \\
\text { branches } \\
\text { per vine }\end{array}$ & $\begin{array}{l}\text { Internodal } \\
\text { length }(\mathrm{cm})\end{array}$ & $\begin{array}{l}\text { Fruit length } \\
\qquad(\mathrm{cm})\end{array}$ & $\begin{array}{c}\text { Fruit } \\
\text { diameter } \\
(\mathbf{c m})\end{array}$ & $\begin{array}{l}\text { Average fruit } \\
\text { weight (g) }\end{array}$ \\
\hline $\begin{array}{l}\text { Phule Green Gold x Improved } \\
\text { Katahi }\end{array}$ & 4.31 & 12.50 & 5.46 & 15.15 & 4.42 & 80.00 \\
\hline Phule Green Gold x Hirkani & 3.62 & 11.92 & 5.41 & 13.21 & 3.75 & 53.92 \\
\hline Phule Green Gold x CO-1 & 4.57 & 12.50 & 7.58 & 19.50 & 4.32 & 89.20 \\
\hline Phule Green Gold x Nakhara Local & 4.28 & 15.50 & 6.45 & 16.00 & 4.13 & 71.60 \\
\hline Phule Green Gold x Thusi & 3.79 & 17.25 & 5.78 & 14.16 & 3.90 & 62.00 \\
\hline Phule Green Gold x Preethi & 4.90 & 14.50 & 7.75 & 19.00 & 5.00 & 102.40 \\
\hline $\begin{array}{l}\text { Phule Green Gold x Pusa Do } \\
\text { Mausami }\end{array}$ & 4.86 & 16.83 & 5.24 & 22.68 & 4.85 & 103.53 \\
\hline Improved Katahi x Hirkani & 3.12 & 11.42 & 5.89 & 10.81 & 3.36 & 40.60 \\
\hline Improved Katahi x CO-1 & 4.30 & 12.00 & 7.26 & 16.00 & 4.35 & 76.21 \\
\hline Improved Katahi x Nakhara Local & 3.60 & 14.83 & 4.53 & 10.06 & 3.58 & 41.30 \\
\hline Improved Katahi x Thusi & 3.65 & 16.33 & 4.10 & 11.55 & 3.85 & 50.00 \\
\hline Improved Katahi x Preethi & 4.51 & 14.33 & 6.80 & 15.65 & 5.20 & 92.30 \\
\hline $\begin{array}{l}\text { Improved Katahi x Pusa Do } \\
\text { Mausami }\end{array}$ & 4.09 & 14.83 & 4.98 & 15.26 & 4.65 & 83.53 \\
\hline Hirkani x CO-1 & 3.38 & 9.00 & 7.17 & 14.60 & 4.06 & 64.50 \\
\hline Hirkani x Nakhara Local & 3.60 & 14.67 & 6.33 & 11.86 & 3.69 & 49.30 \\
\hline Hirkani x Thusi & 3.63 & 19.00 & 4.00 & 11.18 & 3.80 & 45.05 \\
\hline Hirkani x Preethi & 4.56 & 14.08 & 7.55 & 15.46 & 4.78 & 84.13 \\
\hline Hirkani x Pusa Do Mausami & 3.46 & 13.58 & 5.75 & 11.09 & 3.45 & 41.85 \\
\hline CO-1 x Nakhara Local & 3.37 & 7.92 & 6.28 & 10.20 & 3.39 & 40.10 \\
\hline CO-1 x Thusi & 3.30 & 11.92 & 5.50 & 12.02 & 3.84 & 49.05 \\
\hline CO-1 x Preethi & 4.60 & 11.75 & 7.90 & 15.90 & 4.95 & 90.01 \\
\hline CO-1 x Pusa Do Mausami & 3.61 & 10.00 & 6.38 & 12.55 & 3.79 & 58.67 \\
\hline
\end{tabular}




\begin{tabular}{|c|c|c|c|c|c|c|}
\hline Nakhara Local $x$ Thusi & 3.30 & 15.83 & 4.87 & 10.50 & 3.64 & 42.20 \\
\hline Nakhara Local x Preethi & 3.77 & 11.25 & 6.67 & 11.52 & 3.82 & 50.21 \\
\hline Nakhara Local x Pusa Do Mausami & 3.70 & 16.33 & 4.85 & 14.68 & 4.01 & 66.10 \\
\hline Thusi x Preethi & 3.58 & 15.25 & 4.90 & 9.36 & 3.91 & 45.01 \\
\hline Thusi x Pusa Do Mausami & 3.70 & 19.50 & 4.04 & 11.95 & 4.00 & 51.00 \\
\hline Preethi x Pusa Do Mausami & 4.69 & 14.58 & 7.12 & 16.33 & 5.15 & 99.62 \\
\hline Phule Green Gold & 4.08 & 11.83 & 5.85 & 18.04 & 3.86 & 71.35 \\
\hline Improved Katahi & 3.51 & 11.25 & 5.50 & 12.62 & 4.19 & 60.01 \\
\hline Hirkani & 3.97 & 14.17 & 5.63 & 12.15 & 3.83 & 46.00 \\
\hline CO-1 & 4.25 & 8.75 & 6.35 & 14.15 & 3.78 & 63.48 \\
\hline Nakhara Local & 3.12 & 14.17 & 4.50 & 9.29 & 3.32 & 33.58 \\
\hline Thusi & 2.94 & 16.75 & 4.43 & 4.66 & 3.18 & 19.04 \\
\hline Preethi & 4.40 & 9.92 & 6.28 & 13.34 & 4.72 & 83.98 \\
\hline Pusa Do Mausami & 3.01 & 14.00 & 4.25 & 12.40 & 3.94 & 57.65 \\
\hline Mean & 3.87 & 13.62 & 5.81 & 13.47 & 4.07 & 62.74 \\
\hline C.V (\%) & 4.16 & 9.15 & 3.33 & 8.31 & 4.15 & 4.87 \\
\hline S.Em \pm & 0.09 & 0.72 & 0.11 & 0.65 & 0.10 & 1.76 \\
\hline C.D at $5 \%$ & 0.27 & 2.03 & 0.32 & 1.82 & 0.28 & 4.98 \\
\hline Range & $2.94-4.90$ & $7.92-19.50$ & $4.00-7.90$ & $4.66-22.68$ & $3.18-5.20$ & $19.04-103.53$ \\
\hline
\end{tabular}

Table 2 contd....

\begin{tabular}{|l|c|c|c|c|c|}
\hline \multicolumn{1}{|c|}{ Hybrid } & $\begin{array}{c}\text { Number of } \\
\text { fruits per vine }\end{array}$ & $\begin{array}{c}\text { Fruit yield } \\
\text { per vine } \\
(\mathbf{k g})\end{array}$ & $\begin{array}{c}\text { Flesh } \\
\text { thickness } \\
(\mathbf{m m})\end{array}$ & $\begin{array}{c}\text { TSS } \\
\left({ }^{\circ} \mathbf{B r i x}\right)\end{array}$ & $\begin{array}{c}\text { Vitamin C } \\
(\mathbf{m g} / \mathbf{1 0 0 g})\end{array}$ \\
\hline $\begin{array}{l}\text { Phule Green Gold x Improved } \\
\text { Katahi }\end{array}$ & 29.50 & 2.33 & 11.68 & 3.75 & 122.04 \\
\hline
\end{tabular}




\begin{tabular}{|c|c|c|c|c|c|}
\hline Phule Green Gold x Hirkani & 24.00 & 1.16 & 9.16 & 3.37 & 102.04 \\
\hline Phule Green Gold x CO-1 & 28.33 & 2.44 & 11.27 & 4.28 & 115.47 \\
\hline Phule Green Gold x Nakhara Local & 32.00 & 2.23 & 10.40 & 4.00 & 125.10 \\
\hline Phule Green Gold x Thusi & 32.42 & 1.98 & 10.08 & 2.75 & 120.37 \\
\hline Phule Green Gold x Preethi & 30.00 & 3.00 & 13.93 & 4.10 & 138.05 \\
\hline $\begin{array}{l}\text { Phule Green Gold x Pusa Do } \\
\text { Mausami }\end{array}$ & 31.00 & 3.13 & 12.43 & 4.20 & 141.28 \\
\hline Improved Katahi x Hirkani & 23.50 & 0.93 & 8.40 & 3.39 & 90.42 \\
\hline Improved Katahi x CO-1 & 27.92 & 2.09 & 11.35 & 2.43 & 102.15 \\
\hline Improved Katahi x Nakhara Local & 32.50 & 1.23 & 9.14 & 3.60 & 93.02 \\
\hline Improved Katahi x Thusi & 35.75 & 1.70 & 9.80 & 2.27 & 110.15 \\
\hline Improved Katahi x Preethi & 29.17 & 2.62 & 14.92 & 3.11 & 125.73 \\
\hline $\begin{array}{l}\text { Improved Katahi x Pusa Do } \\
\text { Mausami }\end{array}$ & 31.58 & 2.54 & 12.21 & 4.07 & 125.00 \\
\hline Hirkani x CO-1 & 19.17 & 1.32 & 10.28 & 3.66 & 85.02 \\
\hline Hirkani x Nakhara Local & 25.83 & 1.20 & 9.20 & 4.05 & 97.02 \\
\hline Hirkani x Thusi & 39.83 & 1.71 & 9.35 & 2.81 & 100.00 \\
\hline Hirkani x Preethi & 31.00 & 2.40 & 12.34 & 2.52 & 117.00 \\
\hline Hirkani x Pusa Do Mausami & 26.00 & 1.01 & 8.74 & 3.77 & 97.41 \\
\hline CO-1 x Nakhara Local & 18.83 & 0.75 & 8.50 & 4.32 & 79.07 \\
\hline CO-1 x Thusi & 25.08 & 1.15 & 9.70 & 3.77 & 80.40 \\
\hline CO-1 x Preethi & 24.50 & 2.15 & 13.28 & 3.34 & 116.43 \\
\hline CO-1 x Pusa Do Mausami & 17.25 & 0.94 & 9.35 & 4.22 & 97.17 \\
\hline Nakhara Local x Thusi & 28.17 & 1.15 & 9.03 & 3.37 & 86.35 \\
\hline Nakhara Local x Preethi & 24.25 & 1.12 & 9.27 & 3.33 & 94.05 \\
\hline Nakhara Local x Pusa Do Mausami & 28.83 & 1.79 & 10.34 & 4.60 & 95.43 \\
\hline Thusi x Preethi & 24.25 & 1.03 & 9.96 & 3.44 & 92.00 \\
\hline Thusi x Pusa Do Mausami & 41.92 & 2.12 & 10.10 & 3.51 & 126.80 \\
\hline Preethi x Pusa Do Mausami & 28.00 & 2.75 & 14.64 & 4.75 & 132.55 \\
\hline Phule Green Gold & 27.33 & 1.87 & 9.70 & 3.50 & 113.25 \\
\hline Improved Katahi & 29.42 & 1.65 & 10.63 & 2.65 & 105.70 \\
\hline
\end{tabular}


Int.J.Curr.Microbiol.App.Sci (2018) 7(8): 1082-1092

\begin{tabular}{|l|c|c|c|c|c|}
\hline Hirkani & 30.25 & 1.34 & 9.63 & 3.37 & 98.05 \\
\hline CO-1 & 20.50 & 1.21 & 9.40 & 3.71 & 94.30 \\
\hline Nakhara Local & 27.83 & 0.93 & 8.38 & 3.18 & 84.35 \\
\hline Thusi & 36.17 & 0.73 & 7.44 & 2.50 & 86.04 \\
\hline Preethi & 20.17 & 1.57 & 12.39 & 3.67 & 103.18 \\
\hline Pusa Do Mausami & 19.50 & 1.07 & 9.80 & 4.04 & 110.51 \\
\hline Mean & $\mathbf{2 7 . 8 3}$ & $\mathbf{1 . 6 8}$ & $\mathbf{1 0 . 4 5}$ & $\mathbf{3 . 5 4}$ & $\mathbf{1 0 5 . 6 4}$ \\
\hline C.V (\%) & 8.25 & 8.32 & 2.15 & 7.11 & 7.42 \\
\hline S.Em \pm & 1.32 & 0.08 & 0.13 & 0.15 & 4.52 \\
\hline C.D at 5\% & 3.74 & 0.23 & 0.37 & 0.41 & 12.76 \\
\hline Range & $17.25-41.92$ & $0.73-3.13$ & $7.44-14.92$ & $2.27-4.75$ & $79.07-141.28$ \\
\hline
\end{tabular}


Fruit yield per vine is the extreme and the foremost vital trait for any variety or hybrid. For fruit yield per vine the parents, Phule Green Gold (1.87 kg), Improved Katahi (1.65 $\mathrm{kg})$ and Preethi $(1.57 \mathrm{~kg})$ and the hybrids Phule Green Gold x Pusa Do Mausami (3.13 $\mathrm{kg}$ ), Phule Green Gold x Preethi (3.00 kg) and Preethi X Pusa Do Mausami $(2.75 \mathrm{~kg})$ registered higher mean values. Similar results were reported by Jadhav et al., (2009), Rani et al., (2014) and Kumara et al., (2017) in bitter gourd.

With respect to flesh thickness it should be as high as possible. In the present study the parents, Preethi $(12.39 \mathrm{~mm})$ and Improved Katahi $(10.63 \mathrm{~mm})$ recorded maximum pulp thickness whereas among hybrids Improved Katahi x Preethi (14.92 mm), Preethi x Pusa Do Mausami (14.64 mm) and Phule Green Gold x Preethi $(13.93 \mathrm{~mm})$ recorded higher flesh thickness. Similar results were reported by Rani et al., (2014). Among parents, Pusa

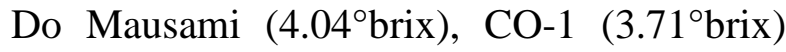
and Preethi $\left(3.67^{\circ}\right.$ brix) recorded maximum TSS content in fruits. Among all the hybrids, it was observed that Preethi $x$ Pusa Do Mausami (4.75 ${ }^{\circ}$ brix), Nakahara Local x Pusa Do Mausami $\left(4.60^{\circ}\right.$ brix) and CO- 1 x Nakahara Local $\left(4.32^{\circ}\right.$ brix) recorded higher TSS content in fruits. Ascorbic acid is a nutritionally important character and the parents Phule Green Gold (113.25 mg), Pusa Do Mausami (110.51 mg) and Improved Katahi (105.70 mg) and the hybrids Phule Green Gold x Pusa Do Mausami (141.28 mg), Phule Green Gold x Preethi (138.05 mg) and Preethi x Pusa Do Mausami (132.55 mg) registered higher magnitudes of ascorbic acid content. These results are in similarity with the results of Rajeswari (1998) and Thangamani and Pugalendhi (2013)..

From the present investigation, it is concluded that parents Phule Green Gold for fruit length, fruit yield per vine and vitamin $\mathrm{C}$ content,
Preethi for vine length, fruit diameter and average fruit weight; Thusi for number of primary branches per vine and number of fruits per vine, were found superior among all the parents. Improved Katahi recorded second position for fruit yield per vine. These may well be utilized in as one of the parent to produce high yielding and superior quality hybrids, as well as in varietal improvement programmes. Further, the best cross combinations (Phule Green Gold x Pusa Do Mausami, Phule Green Gold x Preethi and Preethi $x$ Pusa Do Mausami) may be exploited as commercial hybrids after their stability test as they are not as it high yielder but also possessed good quality characters as per present market demand. Therefore, it is proposed that hybrid breeding approaches may well be more fulfilling since hybrids advocate increased yield and other related traits in comparison with parents.

\section{Acknowledgement}

The authors thank College of Agriculture and AICRP on vegetable crops, OUAT, Bhubaneswar, Odisha, India for providing all the facilities throughout the course of this research work and the corresponding author is gratitude to Department of Science \& Technology (DST), Government of India, New Delhi for providing Inspire Fellowship for the research work.

\section{References}

Ahmed, I., M.S. Lakhani, M. Gillett, A. John and Raza, H. 2001. Hypotriglyceridemic and hypocholesterolemic effects of antidiabetic Momordica charantia (karela) fruit extract in streptozotocin-induced diabetic rats. Diabetes Research and Clinical Practice. 51: 155-161.

Barron, D., M. Kaouadji and Mariotte, A.M. 1982. Etude comparative de deux 
cucurbitacees a usage medicinal. Planta Medica. 46: 184-186.

Basch, E., S. Gabardi and Ulbrich, C. 2003. Bitter melon (Momordica charantia): A review of efficiency and safety. American Journal of Health-System Phermacy. 60: 356-359.

Chen, Q., L. Laureen, L. Chan and Li, E.T. 2003. Bitter melon (Momordica charantia) reduces adiposity, lowers serum insulin and normalizes glucose tolerance in rats fed a high fat diet. Journal of Nutrition. 133:1088-1093.

Devadass, V.S. 1993. Genetic studies on fruit and seed yield and quality in bitter gourd (Momordica charantia L.). Ph.D. (Hort.) Thesis, Tamil Nadu Agricultural University, Coimbatore.

Dey, S.S., T.K. Behera, A. Pal and Munshi, A.D. 2005. Correlation and path coefficient analysis in bitter gourd (Momordica charantia L.). Vegetable Science. 32:173-176.

Ganguly, C., S. De and Das, S. 2000. Prevention of carcinogen induced mouse skin papilloma by whole fruit aqueous extract of Momordica charantia. European Journal of Cancer Prevention. 9: 283-288.

Gurbuz, I., C. Akyuz, E. Yesilada and Bilge, S. 2000. Anti-ulcerogenic effect of Momordica charantia L. fruits on various ulcer models in rats. Journal of Ethnopharmacology. 71: 77-82.

Harika, M., V.D. Gasti, T. Shantappa, R. Mulge, A.M. Shirol, A.B. Mastiholi and Kulkarni, M.S. 2012. Evaluation of bottle gourd genotypes [Lagenaria siceraria (Mol.) Standl.] for various horticultural characters. Karnataka Journal of Agricultural Sciences 25(2): 241-244.

Jadhav, K.A., B.V. Garad, S.S. Dahmal, D.B. Kashirsagar, B.T. Patil and K.G. Shinde. 2009. Heterosis in bitter gourd (Momordica charantia L.). Agricultural
Science Digest. 29 (1): 7-11.

Jayasooriya, A.P., M. Sakono, C. Yukizaki, M. Kawano, K. Yamamoto and Fukuda, N. 2000. Effects of Momordica charantia powder on serum glucose levels and various lipid parameters in rats fed with cholesterol-free and cholesterolenriched diets. Journal of Ethnopharmacology. 72: 331-336.

Jeffrey, C. 1980. A review of the Cucurbitaceae. Botanical Journal of the Linnean Society. 81:233-247.

Kumara, B.S, T.B. Puttaraju and Pavithra, H.B. 2017. Evaluation of Bitter Gourd (Momordica charantia L.) Hybrids under Eastern Dry Zone of Karnataka, India. International Journal of Current Microbiology and Applied Sciences 6(11):1931-1939.

Laxuman., S.A. Patil, P.M. Salimath, P.R. Dharmatti, A.S. Byadgi and Nirmalayenagi. 2012. Heterosis and combining ability analysis for productivity traits in bitter gourd (Momordica charantia L.). Karnataka Journal Agricultural Science 25(1): 913.

Lee, H.S., P.L. Huang, P.L. Huang, A.S. Bourinbaiar, H.C. Chen and Kung, H.F. 1995. Inhibition of the integrase of human immuno- deficiency virus (HIV) type 1 by anti-HIV plant proteins MAP30 and GAP31. Proceedings of National Academy of Science. (USA). 92: 8818-8822.

Leung, K.C., Z.Q. Meng and Ho, W.K.K. 1997. Antigenic determination fragments of $\alpha$ - momorcharin. Biochimica et Biophysica Acta. 1336: 419-424.

Nerurkar, P.V., Y.K. Lee, E.H. Linden, S Lim, L. Pearson, J. Frank and Nerurkar, V.R. 2006. Lipid lowering effects of Momordica charantia (Bitter Melon) in HIV-1-protease inhibitor-treated human hepatoma cells, HepG2. British Journal 
of Pharmacology. 148: 1156-1164.

NHB database. (2015-16). Indian Horticulture Database, National Horticultural Board, Gurgoan.

Okabe, H., Y. Miyahara, T. Yamauchi, K. Mirahara and Kawasaki, T. 1982. Studies on the constituents of Momordica charantia L. Isolation and characterization of momordicosides A and B, glycosides of a pentahydroxycucurbitane triterpene. Chemical and Pharmaceutical Bulletin. 28: 2753-2762.

Okabe, H., Y. Miyahara, T. Yamauchi, K. Mirahara and Kawasaki, T. 1980. Studies on the constituents of Momordica charantia L. I. Isolation and characterization of momordicosides A and B, glycosides of a pentahydroxycucurbitane triterpene. Chemistry and Phermacology Bulletine 28: 2753-2762.

Panse, V.G. and Sukhatme, P.V. 1957. Statistical Methods for Agricultural Workers. Indian Council of Agricultural Research, New Delhi. p. 97.

Rajeswari, K.S. 1998 Genetic studies in bitter gourd (Momordica charantia L.) through diallel analysis. M.Sc. (Hort.) Thesis, Tamil Nadu Agricultural University, Coimbatore.

Rani, K.R., Ch.S. Raju and Reddy, K.R. 2014. A study on heterosisi for yield and earliness in Bitter gourd. Indian Journal of Scientific Research and Technology 2(3):89-97

Sreejayan, and Rao, M.N.A. 1991. Oxygen free radical scavenging activity of Momordica charntia fruits. Fitoterpeda. 62: 344-46.

Sundaram, V. 2006. Studies on genetics of yield and yield components in bitter gourd (Momordica charantia L.) under salinity. Ph.D (Hort.) Thesis, Tamil Nadu Agricultural University, Coimbatore.

Taylor, L. 2002. Technical Data Report for Bitter melon (Momordica charantia). In Herbal Secrets of the Rainforest. 2nd $e d$, Sage Press Inc.

Thangamani, C. and Pugalendhi, L. 2013. Heterosis Studies in Bitter Gourd for Yield and Related Characters. International Journal of Vegetable Science. 19: 109-125.

Yeh, G.Y., D.M. Eisenber, T.J. Kaptchuk and Phillips, R.S. 2003. Systematic review of herbs and dietary supplements for glycemic control in diabetes. Diabetes Care. 26: 1277-1294.

Yesilada, E., I. Gurbuz and Silabata, H. 1999. Screening of Turkis-ulcerogenic folk remedies for anti- Helicobacter pyroli activity. Journal

of Ethnophermacology. 66: 289-293

\section{How to cite this article:}

Mallikarjunarao, K., A.K. Das, A. Nandi, B. Baisakh, G.S. Sahu and Tripathy, P. 2018. Evaluation of Parents and Hybrids for Yield and Quality characters in Bitter Gourd (Momordica charantia L.). Int.J.Curr.Microbiol.App.Sci. 7(08): 1082-1092. doi: https://doi.org/10.20546/ijcmas.2018.708.123 